\title{
The Acute Effects of Yoga on Executive Function
}

\author{
Neha Gothe, Matthew B. Pontifex, Charles Hillman, and Edward McAuley
}

\begin{abstract}
Background: Despite an increase in the prevalence of yoga exercise, research focusing on the relationship between yoga exercise and cognition is limited. The purpose of this study was to examine the effects of an acute yoga exercise session, relative to aerobic exercise, on cognitive performance. Methods: A repeated measures design was employed where 30 female college-aged participants (Mean age $=20.07, \mathrm{SD}=1.95$ ) completed 3 counterbalanced testing sessions: a yoga exercise session, an aerobic exercise session, and a baseline assessment. The flanker and n-back tasks were used to measure cognitive performance. Results: Results showed that cognitive performance after the yoga exercise bout was significantly superior (ie, shorter reaction times, increased accuracy) as compared with the aerobic and baseline conditions for both inhibition and working memory tasks. The aerobic and baseline performance was not significantly different, contradicting some of the previous findings in the acute aerobic exercise and cognition literature. Conclusion: These findings are discussed relative to the need to explore the effects of other nontraditional modes of exercise such as yoga on cognition and the importance of time elapsed between the cessation of the exercise bout and the initiation of cognitive assessments in improving task performance.
\end{abstract}

Keywords: aerobic exercise, nontraditional exercise, interference control, n-back task

The relationship between exercise and cognitive performance is a highly topical area of scientific inquiry. ${ }^{1}$ Although the majority of this work examines the effects of chronic exercise participation on cognition, there has been an increasing interest in the potential transient effects of acute bouts of exercise. Several reviews and meta-analyses have suggested that participation in acute aerobic activity exerts a positive influence on cognitive function, and in particular, executive function. ${ }^{2-5}$ Executive function refers to a subset of goal-directed processes (ie, planning, scheduling, working memory, task coordination, cognitive flexibility, abstract thinking, etc.) involved in the intentional component of environmental interaction. Both chronic physical activity ${ }^{6}$ and single acute bouts of exercise ${ }^{7}$ have been found to have positive influences on cognition, with disproportionately greater effects for tasks that require extensive amounts of executive control. In particular, one aspect of executive control that has garnered a great deal of interest is inhibitory control, which refers to the ability to manage and direct attention within the stimulus environment by ignoring irrelevant information and maintaining focus on relevant items. Hillman et $\mathrm{al}^{7}$ used a modified flanker task to manipulate inhibitory control, and demonstrated that adults are able to better engage and classify information

The authors are with the Dept of Kinesiology and Community Health, University of Illinois at Urbana-Champaign. Pontifex is now at the Dept of Kinesiology, Michigan State University. in their stimulus environment and process this information more quickly following acute aerobic exercise. The majority of the acute exercise and cognition research has focused on aerobic exercise with relatively fewer studies investigating the effects of other modes of activity. It is difficult to compare or generalize the findings of these studies, given the different protocols and modes of exercise used, and various tasks used to assess unique aspects of cognition. Although there is growing interest in acute exercise and cognition research, the primary focus on aerobic exercise effects makes it difficult to generalize the findings to other nontraditional forms of exercise. Accordingly researchers need to investigate the unique acute effects of other nontraditional modes of exercise such as yoga, tai-chi, and other forms of martial arts. It is known that the cardiovascular and metabolic demands of aerobic and resistance exercises are inherently different. Many of the nontraditional forms of exercise have mindbody components which may differently affect physical and mental processes.

Ross and Thomas ${ }^{8}$ reviewed studies comparing the effects of yoga, a mind-body-based exercise; and aerobic exercise and concluded that in both healthy and diseased populations yoga may be as effective, or better than, aerobic exercise at improving a variety of health-related outcome measures. Yoga is an ancient Indian science and way of life that includes the practice of specific postures, regulated breathing, and meditation. ${ }^{9}$ The technique involves an active attentional or mindfulness component but its potential benefits have not been thoroughly 
explored. Despite an increase in interest pertaining to the health benefits of yoga practice, research focusing on the relationship between yoga practice and cognition is limited. Sarang and Telles ${ }^{10}$ evaluated performance on a 6-letter cancellation task in males (age 18-48 years) immediately before and after 2 yoga-based relaxation techniques and a control session of equal duration. They used cyclic meditation and supine rest and found that the net scores were significantly higher after both practices. In another study using the 6-letter cancellation task, Telles et $\mathrm{al}^{11}$ reported improvement in cancellation scores (either total errors or net scores) after engaging in a yoga breathing technique characterized by forceful exhalation and high-frequency breathing. Canter and Ernst ${ }^{12}$ conducted a systematic review of 10 randomized controlled trials to evaluate the effect of transcendental meditation on cognitive function. Four trials reported large positive effects on cognitive function; 4 provided only weak evidence for a positive effect and 2 showed no effect. Analogous to the aerobic literature, there is clearly mixed evidence regarding acute yoga benefits and cognitive function. Given the various forms and postures of yoga, it is not surprising that these results are equivocal.

The primary focus of exercise and executive function studies has been inhibitory control. However, there is a need to explore other critical executive processes such as working memory. Pontifex et $\mathrm{al}^{13}$ showed that participants' reaction time on a modified Sternberg working memory task improved immediately and 30 minutes after acute aerobic exercise relative to the preexercise baseline. They observed no such effects after resistance exercise or seated rest. In the current study, we used the n-back task as a measure of working memory, one aspect of executive control that is responsible for continuous encoding, temporary storage and manipulation of information necessary to execute complex cognitive tasks. The n-back task is one of the most popular experimental paradigms for cognition and functional neuroimaging studies of working memory. ${ }^{14} \mathrm{~A}$ modified flanker task was used in conjunction with the n-back as it has been used in acute exercise studies to manipulate inhibitory control.

In the current study we employed a repeated measures design to investigate the immediate effect of exercise on cognitive performance in young adults. More specifically, we compared single bouts of yoga and aerobic exercise to assess whether yoga practice improved cognitive performance beyond aerobic exercise. The flanker ${ }^{15-17}$ and n-back ${ }^{18,19}$ tasks were used in this study as these have been used in prior research and would enable us to compare the results with previous studies. It was hypothesized that task performance on these executive function measures would be enhanced by both exercise modalities when compared with baseline performance. Such findings would provide additional support for the existing acute-aerobic exercise literature as well as compare the magnitude of acute yoga effects in relation to aerobic exercise.

\section{Methods}

\section{Participants}

Thirty female undergraduate students (mean age $=20.1$ \pm 2.0 years) from the University of Illinois at UrbanaChampaign were recruited to participate in this study. An informed consent approved by the Institutional Review Board of the University of Illinois at Urbana-Champaign was completed by all the participants. In addition, the Physical Activity Readiness Questionnaire (PAR-Q) was used as a screening tool to detect potential risk factors. All participants reported being free from any conditions on the PAR-Q that might be exacerbated by acute exercise participation. We also excluded participants who were regular practitioners of yoga or other mind-body based exercises (eg, tai-chi, martial arts, etc.) to avoid confounding with the yoga intervention.

\section{Measures}

Exercise History. An exercise history questionnaire was used to assess the frequency, duration and intensity of physical activity over the past 6 months. Participants were asked, "In the last 6 months, on average, how many days a week have you spent 30 minutes or more in moderate to strenuous exercise." Participants responded by checking the number of days/week ranging from $0-7$. Duration was assessed by asking "If you have been exercising, on average how long has each exercise session been?" Participants responded by checking one of these choices: "less than 5 minutes, 5-19 minutes, 20-29 minutes, more than 30 minutes." A total of 17 participants met the CDC guidelines of 150 minutes/ week or more of aerobic activity and were categorized as being 'active.'

Submaximal Fitness Assessment. Maximal aerobic capacity $\left(\mathrm{VO}_{2} \mathrm{max}\right)$ was estimated by participant's performance on a modified Balke submaximal treadmill test. ${ }^{20}$ The protocol involved jogging/running on a treadmill at a constant speed with increasing grade increments of $1 \%$ every minute until the participant reached his/her $85 \%$ predicted max heart rate (HR). A Polar HR monitor (Model A1; Polar Electro, Kempele, Finland) measured HR throughout the test, and a rating of perceived exertion (RPE) ${ }^{21}$ was taken at the end of every minute. The mean (SD) estimated $\mathrm{VO}_{2}$ max for all participants was $35.9(4.8) \mathrm{ml} / \mathrm{kg} / \mathrm{min}$.

Cognitive Measures. We used 2 measures of cognitive function to assess working memory and inhibitory control. Descriptions of these tests follow.

The Flanker Task. A modified flanker task ${ }^{15-17}$ which incorporated arrays of arrows was used to manipulate inhibitory control. The congruent trials consisted of the target arrow being flanked by other arrows that faced the same direction (eg, $<<<<<$ or $>>>>>)$. The incongruent trials consisted of the target arrow being flanked by other 
arrows that faced the opposite directions (eg, $<<><<$ or $>><>>$ ). Participants were instructed to respond as quickly and as accurately as possible with a left button press when the target arrow (regardless of condition) faced to the left (eg, ' $<$ ') and a right button press when the target arrow faced to the right (eg, '>'). During each of the 3 visits, 1 block of 200 trials, randomized across task conditions was presented. The block consisted of 100 congruent and 100 incongruent trials with left and right target arrows occurring with equal probability. The stimuli were $3 \mathrm{~cm}$ tall white arrows, which were presented focally for $80 \mathrm{~ms}$ on a black background with a variable intertrial interval of 1100,1300 , or 1500 ms. Total task duration was approximately $6 \mathrm{~min}$.

The $\mathbf{N}$-back Task. A modified serial n-back task ${ }^{18,19}$ involved 3 consecutive phases called the 0-back, 1-back, and the 2-back. Each phase required the participants to discriminate between 5 distinct shapes. Each shape was represented by a color with blue circles, green triangles, purple stars, red squares, and yellow crosses as the stimuli. In the 0-back condition, participants were instructed to respond as quickly and accurately as possible with a right button press when the yellow cross appeared and with a left button press when any other shape appeared. In the 1-back and 2-back conditions, participants were instructed to respond as quickly and accurately as possible with a right button press if the current shape was the same as the previous trial for the 1-back condition, and 2 trials previous for the 2-back condition. If the current shape was different from the previous trial in the 1-back condition, or from 2 trials previous in the 2-back condition, participants were instructed to make a left button press. During each of the 3 visits, 1 block of 80 trials, randomized across task conditions, was presented for each $\mathrm{n}$-back condition with equally probable presentation of each stimulus ( 16 trials for each shape). All stimuli were $3 \mathrm{~cm}$ tall, presented 1 at a time on a computer screen with a black background for a duration of $250 \mathrm{~ms}$ with a fixed $3000 \mathrm{~ms}$ intertrial interval. The total time to complete all 3 phases of this task was approximately 18 minutes.

\section{Procedure}

A within-subjects repeated measures design had participants visit the laboratory on 3 separate days (mean $=$ $4.1 \pm 3.05$ days between sessions) in which they had not previously participated in any form of physical activity. On the first visit to the laboratory, participants provided written informed consent, completed the PAR-Q and an exercise history questionnaire. The order of the baseline, aerobic exercise, and yoga exercise conditions were counterbalanced across participants to minimize any effects of order or practice. During the baseline session, participants completed the flanker and n-back tasks. Following completion of these cognitive tests, participants performed the submaximal treadmill test, were given a cool down period, and waited for their heart rate to return to within 10 beats per minute of their resting HR before leaving the laboratory.

Participants wore a Polar heart rate monitor (Model A1, Polar Electro, Finland) throughout the exercise sessions, and their resting HR (before beginning the session) and exercise HR (average after 20 minutes of exercise) were recorded by the exercise leader. All the exercise sessions took place individually, one-on-one with the exercise leader. For the yoga visit, a 20-minute progression of seated, standing, and supine yoga postures (see Table 2) was led by an exercise leader trained in Hatha Yoga practice. The postures included isometric contraction and relaxation of different muscle groups in conjunction with regulated breathing, and concluded with a meditative deep breathing posture. The participants mirrored the exercise leader and focused on performing the postures mindfully, attending to their breathing and being aware of the physical posture and muscle movements. Each posture (1-8) listed in Table 2 was performed twice and held up to 30 seconds. For the aerobic exercise visit, participants were supervised by the exercise leader and self-selected the speed and incline combination to stay within $60 \%-70 \%$ of their estimated HR max (mean = $154.0 \pm 9.16 \mathrm{bpm})$ as they exercised on a motor-driven treadmill (see Table 1). This range was chosen to replicate previous findings that have shown improved cognitive performance in response to this intensity. ${ }^{13}$ Immediately following completion of the exercise conditions, participants were escorted to the cognitive testing room and provided task instructions for the cognitive tasks. The n-back and flanker tasks were administered in a counterbalanced order across participants but fixed within each participant across exercise modalities. Each of the 3 sessions were conducted in the same exercise room and were performed individually by the participants. The mean time elapsed before the beginning of cognitive testing, the duration of the visits and cognitive assessments is shown in Table 1.

\section{Data Analysis}

Before hypothesis testing, preliminary analyses were conducted to examine the order in which the exercise sessions and cognitive tasks occurred to ensure that the observed effects were not due to the specific order in which participants received the various conditions. No significant differences in session order $[F(5,24) \leq 1.69$, $P \geq .18]$ or cognitive task order [ $t(28) \leq 1.04, P \geq .308]$ were observed for any of the reaction time (RT) or accuracy variables. In addition, we examined the potential for activity level moderating cognitive responses and found no significant mean differences $[t(28) \leq 1.58, P \geq .13]$ when comparing the 'active' versus 'inactive' (based on CDC guidelines) participants.

Primary analyses used repeated-measures ANOVAs with a family-wise alpha level set at 0.05 to determine whether the 3 conditions (baseline, aerobic, yoga) resulted in differential task performance. Specifically, analyses of task performance (ie, RT and response accuracy) 
Table 1 Duration of Visits and Mean Heart Rates for the Exercise Conditions

\begin{tabular}{|c|c|c|c|}
\hline & Baseline mean (SD) & Aerobic mean (SD) & Yoga mean (SD) \\
\hline Total duration of the visit (minutes) & $52.8^{\mathrm{a}, \mathrm{b}}(3.9)$ & $56.97 \mathrm{a}(5.3)$ & $57.41^{\mathrm{b}}(4.8)$ \\
\hline Time elapsed before cognitive testing (minutes) & N/A & $4.28(1.6)$ & $4.24(1.5)$ \\
\hline Duration of the cognitive testing (minutes) & $36.82(3.9)$ & $36.97(5.3)$ & $37.41(4.8)$ \\
\hline Resting heart rate (bpm) & N/A & $77(7.6)$ & $80.43(10.6)$ \\
\hline Exercise heart rate $(\mathrm{bpm})^{\mathrm{b}}$ & N/A & $154.03(9.2)$ & $77.50(14.19)$ \\
\hline Mean rating of perceived exertion ${ }^{\mathrm{b}}$ & N/A & $13.10(2.1)$ & $8.73(1.9)$ \\
\hline
\end{tabular}

${ }_{\mathrm{a}, \mathrm{b}}$ Indicates a significant mean difference between conditions $(P<.01)$.

Abbreviations: bpm, beats per minute.

Table 2 Description of the Yoga Exercise Session

\begin{tabular}{lll}
\hline Order & Position name & Duration \\
\hline 1 & Uttanasana-Standing Forward Bend & 1 minute \\
2 & Vrikshasana-Tree Pose & 1 minute \\
3 & Trikonasana-Triangle Pose & 2 minutes \\
4 & Parivrtta Trikonasana-Reverse Triangle Pose & 2 minutes \\
5 & Adho Mukha Shvanasana-Downward Facing Dog & 2 minutes \\
6 & Ustrasana-Easy Camel Pose & 2 minutes \\
7 & Shashankasana-Hare Pose & 2 minutes \\
8 & Suryanamaskar-Salute to the Sun & 4 minutes \\
9 & Padmasana Pranayama-Deep Breathing in Lotus Pose & 4 minutes \\
\hline
\end{tabular}

were conducted using multivariate repeated-measures ANOVA. Analysis of the flanker task was conducted using a 3 (Condition: Baseline, Aerobic Exercise, Yoga Exercise) $\times 2$ (Congruency: Congruent, Incongruent) multivariate repeated-measures ANOVA whereas, for the n-back task a 3 (Condition: Baseline, Aerobic Exercise, Yoga Exercise) $\times 3$ (Memory Load: 0-back, 1-back, 2-back) model was tested. Post hoc comparisons were conducted using Bonferroni adjustment.

\section{Results}

\section{Flanker Task}

RT. There was no significant main effect of condition $F(2,28)=.77 P=.47$, partial $\eta^{2}=.05$. However, congruency was significant $F(1,29)=339.94, P<.001$, partial $\eta^{2}=.92$ indicating that RTs for congruent trials were significantly shorter than incongruent trials. No condition $\times$ congruency interaction was observed $F(2,28)=.31, P$ $=.74$, partial $\eta^{2}=.02$.

Response Accuracy. Analyses revealed a main effect of condition, $F(2,28)=5.69, P=.008$, partial $\eta^{2}=.29$, and congruency, $F(1,29)=41.11, P=.000$, partial $\eta^{2}=$ .59 , which was superseded by a condition $\times$ congruency interaction, $F(2,28)=9.22, P=.001$, partial $\eta^{2}=.40$. Post hoc analyses of this interaction examined condition within congruency and revealed no differences for the congruent trials. However, accuracy was significantly greater in the yoga condition as compared with both the aerobic condition $[t(29)=4.27, P<.001]$ and the baseline condition $[t(29)=3.01, P<.016]$ for the incongruent trials. The pairwise comparison of the aerobic condition with the baseline condition was nonsignificant $[t(29)=$ $.675, P<.51]$.

\section{N-back Task}

$\boldsymbol{R T}$. There was a significant main effect of condition $F(2,28)=4.94, P=.02$, partial $\eta^{2}=.26$, and memory load, $F(2,28)=23.10, P<.001$, partial $\eta^{2}=.62$, which was superseded by a condition $\times$ memory load interaction, $F(4,26)=3.90, P=.01$, partial $\eta^{2}=.38$. Decomposition of this interaction by examining condition within memory load revealed no significant differences between the 3 conditions $[t(29) \leq .12, P \geq .99]$ for the 0 -back, however yoga RT was significantly shorter than the aerobic RT $[t(29)=2.85, P<.025]$ for the 1 -back task. A similar pattern was seen for the 2-back task where yoga RT was significantly shorter than the aerobic RT $[t(29)=3.24, P$ $<.01]$. Interestingly, the mean RTs were not significantly 
different between the yoga and baseline conditions for the 1-back $[t(29)=1.9, P=.214]$ or the 2 -back tasks $[t(29)$ $=1.95, P<.19]$.

Response Accuracy. There was a significant main effect of condition $F(2,28)=20.06, P<.001$, partial $\eta^{2}$ $=.59$ and memory load $F(2,28)=15.61, P=.000$, partial $\eta^{2}=.53$ with decreasing accuracy as the load increased from 0 to back to 1-back to 2-back. Post hoc analyses of the condition main effect revealed that accuracy for the yoga condition was significantly greater when compared with the aerobic condition $[t(29)=5.01, P=.003]$ as well as baseline $[t(29)=6.07, P=.02]$ across the 3 memory loads. The pairwise comparison of the aerobic condition with the baseline condition was nonsignificant $[t(29)=$ $.66, P<.52]$. No condition $\times$ memory load interaction was observed $F(4,26)=.47, P=.76$, partial $\eta^{2}=.07$.

The means and standard deviations for the flanker and n-back tasks across the study conditions are presented in Table 3 and Figures 1 and 2.

Table 3 Reaction Time and Accuracy for the Cognitive Tasks Across Conditions

\begin{tabular}{lccc}
\hline & Baseline mean (SD) & Aerobic mean (SD) & Yoga mean (SD) \\
\hline Flanker (congruent) mean RT & $366.97(49.90)$ & $372.02(43.67)$ & $373.40(53.70)$ \\
Accuracy & $91.30(9.95)$ & $91.03(10.22)$ & $93.87(8.76)$ \\
Flanker (incongruent) mean RT & $413.19(53.87)$ & $421.41(53.28)$ & $419.03(60.46)$ \\
Accuracy & $84.37(12.61)^{\mathrm{a}}$ & $83.37(12.27)^{\mathrm{b}}$ & $90.27(10.63)^{\mathrm{a}, \mathrm{b}}$ \\
0-back mean RT & $415.72(90.68)$ & $415.52(84.85)$ & $414.07(87.64)$ \\
Accuracy & $89.58(15.07)^{\mathrm{a}}$ & $89.40(12.44)^{\mathrm{b}}$ & $96.04(8.45)^{\mathrm{a}, \mathrm{b}}$ \\
1-back mean RT & $485.97(107.21)^{\mathrm{a}}$ & $494.86(119.28)^{\mathrm{b}}$ & $458.34(109.11)^{\mathrm{a}, \mathrm{b}}$ \\
Accuracy & $85.17(11.26)^{\mathrm{a}}$ & $83.23(13.92)^{\mathrm{b}}$ & $93.17(7.93)^{\mathrm{a}, \mathrm{b}}$ \\
2-back mean RT & $602.43(215.24)^{\mathrm{a}}$ & $643.47(216.86)^{\mathrm{b}}$ & $551.08(163.62)^{\mathrm{a}, \mathrm{b}}$ \\
Accuracy & $78(12.91)^{\mathrm{a}}$ & $77.17(15.41)^{\mathrm{b}}$ & $86.67(14.34)^{\mathrm{a}, \mathrm{b}}$ \\
\hline
\end{tabular}

Note. Mean RT in milliseconds, accuracy in \% correct response; ${ }^{\mathrm{a}, \mathrm{b}}$ indicates a significant mean difference between conditions $(P<.01)$.
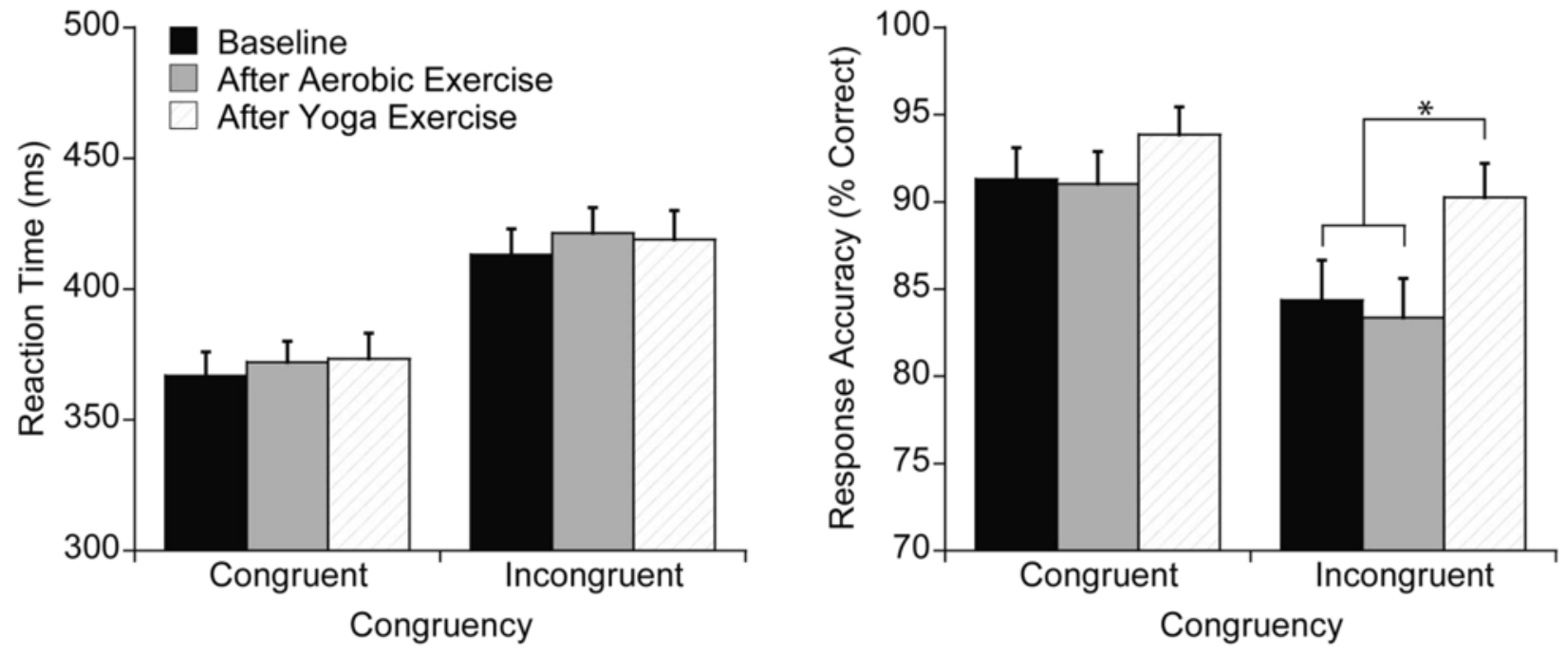

Figure 1 - Reaction time (ms) and accuracy (percent correct) on the flanker task for the 3 conditions. 

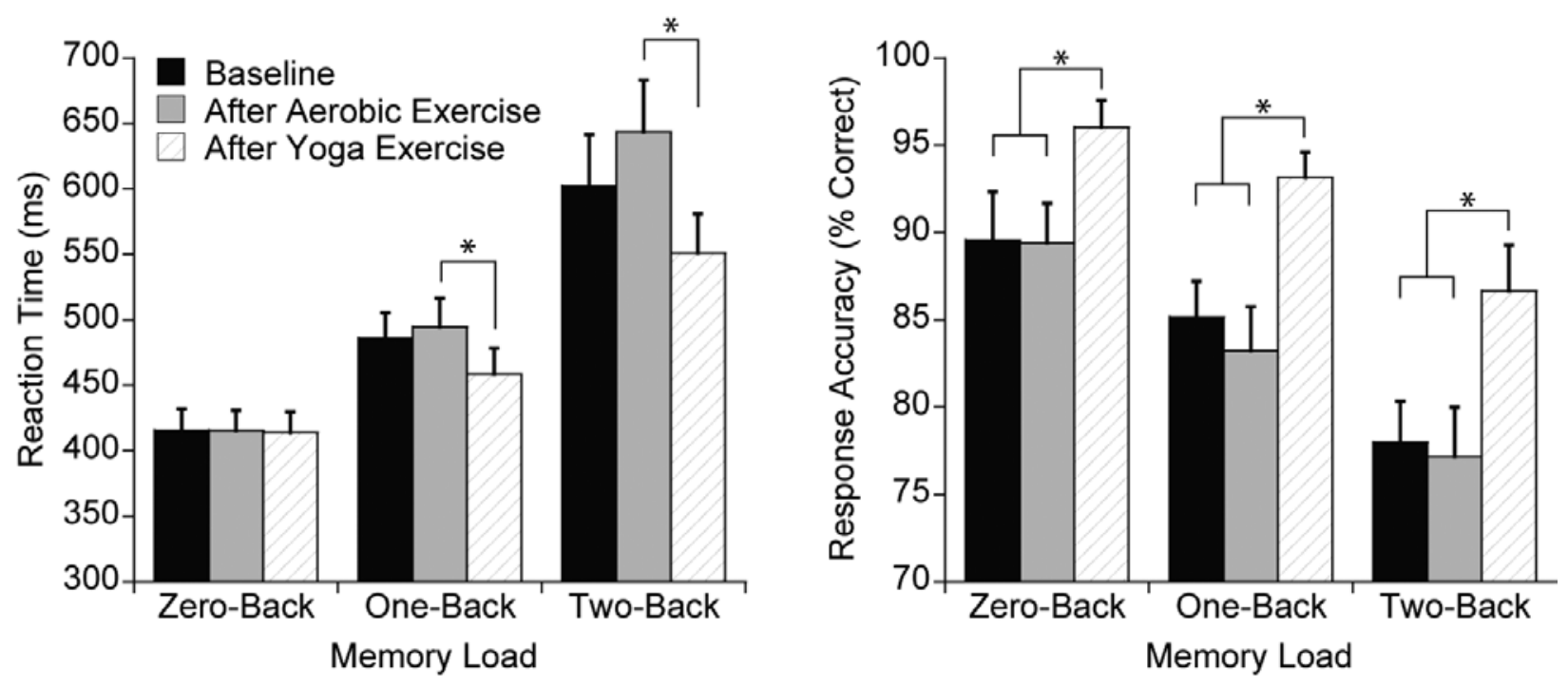

Figure 2 - Reaction time (ms) and accuracy (percent correct) on the n-back task for the 3 conditions.

\section{Discussion}

The purpose of this study was to compare the immediate effects of an acute bout of yoga and aerobic exercise on executive function tests of inhibition and working memory. The current findings indicated that the reaction times were shorter and the accuracy was significantly greater after an acute bout of yoga for tasks requiring greater amounts of executive control, indicating improvements in inhibition and working memory. In contrast to previous research, similar effects were not observed after an acute aerobic exercise bout at $60 \%-70 \%$ of $\mathrm{HR}_{\max }{ }^{13}$ or at $83 \%-84 \%$ of $\mathrm{HR}_{\max } .{ }^{7}$ In the current study, performance following the aerobic exercise bout was comparable to the baseline performance of the participants for some of the task conditions (see Table 2 for means).

Contrary to our a priori hypothesis, improvements in task performance were not observed following an acute bout of aerobic exercise. There are 3 possible reasons for this equivocality. Firstly, researchers have used the terms "immediate" and "prolonged" to assess acute exercise effects on cognition. ${ }^{22}$ However, details of the time elapsed between the end of the exercise bout and beginning of the cognitive assessments is rarely reported making interpretation of the term "immediate" difficult. Some of these studies have employed neuroimaging techniques such as $\mathrm{EEG}^{7,17}$ which require extensive prepping and assembly before beginning the cognitive assessments. The extent to which these findings can be considered true "immediate" exercise effects is debatable, given that testing began approximately 48 min after the cessation of the acute bout. ${ }^{7}$ Given this drawback, the current findings add to the acute-exercise literature by investigating the true "immediate" effects observed within 5 minutes of the cessation of the exercise bout (see Table 1 for cognitive testing duration). However, the results of this study disagree with the true immediate effects found by Pontifex et $\mathrm{al}^{13}$ who used a modified Sternberg task to assess working memory. Working memory is composed of 2 segregated components: maintenance and manipulation. Maintenance has been defined as transferring, maintaining (including rehearsal), and matching of information in working memory ${ }^{23}$ whereas manipulation refers to the additional reorganization or updating of each memory set. The dissimilarity in the results may be due to the different tasks used in these studies. The n-back is a continuous performance task that appears to tap the manipulation component, whereas the Sternberg task may only reflect maintenance processes associated with working memory. The third possible explanation for the contradictory results may relate to gender differences in the samples tested. Studies that report enhanced cognitive performance have had small but an equal number of male and female participants (eg, $\mathrm{n}=10$ males and $10 \mathrm{females}^{7}$ or a disproportionate ratio of male-to-female (12:9). ${ }^{17}$ Although such demographics are statistically controlled in the analyses, it is plausible that the improved cognitive performance is a gender specific phenomenon or the effects are disproportionate for the 2 genders. This study, to our knowledge, is the first to have assessed the acute exercise effects on a homogenous sample of females.

Yoga is a commonly practiced mind-body approach that has components centering on meditation, breathing, and activity or postures. There appear to be at least 2 mechanisms by which the practice of yoga or exercise improves cognitive ability. Lowered mood is associated with declines in cognitive function and Hatha yoga has been reported to produce improvements in mood comparable to aerobic exercise. ${ }^{24,25}$ Additionally, the practice of yoga emphasizes body awareness and involves focusing 
one's attention on breathing or specific muscles or parts of body; therefore it is possible that yoga may improve more general attentional abilities. Attentional focus is a major aspect of yoga practice. It produces similar effects as relaxation in that it tends to promote self-control, attention and concentration, self-efficacy, body awareness and stress reduction. ${ }^{26}$ Such effects may explain why higher accuracy was recorded on the more demanding task conditions like the incongruent flanker, even though the RT was not significantly different across conditions. Anecdotal participant evaluation poststudy reported feelings of calmness and relaxation after the yoga session, whereas some reported feeling tired following the aerobic session. Yoga practice has been reported to help reduce anxiety based on a reduction in psychological arousal ${ }^{27}$ and some studies have shown that anxiety affects performance on tasks requiring attention. ${ }^{28}$ Sarang and Telles ${ }^{10}$ speculated that anxiety reduction was the likely basis for better performance in their study. Future research examining acute yoga effects on cognition should employ additional physiological assessments to further investigate such hypotheses. It is also possible that participant expectancies from the yoga intervention may have led to their improved performance on the cognitive tasks. Given that these participants did not perform yoga routinely, the novelty and instruction during the yoga session and the mind-body nature of this exercise may have resulted in motivational and attentional enhancements during the cognitive tasks resulting in improved performance. However, it is difficult to determine expectancy effects for physical activity based interventions since the participants' themselves perform the physical activity and are therefore not blinded to the intervention. This mechanism may operate for any form of physical activity leading to not only cognitive improvements but also positive changes in other psychological outcomes that are assessed in physical activity based studies. While these mechanisms can only be speculated, they demand further inquiry.

In spite of the strengths of our study, we acknowledge a number of limitations. First, our sample was small and consisted entirely of female college students. It remains to be seen whether males within this age group would also show an improved cognitive performance after an acute yoga session. Second, we had the participants complete an $85 \%$ submaximal test to assess their aerobic capacity. Although a graded maximal exercise test is the gold standard for cardiovascular fitness assessment, we employed a submaximal fitness test for 2 reasons: 1) it was not a primary outcome of this study; and 2) the rigorous methodology of a repeated measures design made it less important of an issue to obtain the best possible measurement of fitness, also reducing participant burden.

In summary, our findings suggest that specific types of acute exercise differentially influence executive control processes. Participation in a 20-minute yoga session resulted in superior cognitive performance compared with acute aerobic activity and baseline assessment. Given the cardiorespiratory and metabolic differences between aerobic and yoga exercise, future research should address the relationship between yoga, cerebral blood flow, and cognition to provide additional insight into the relationship between cognition and exercise behavior. It is unknown at this time if these results are related to the differential cardiovascular and metabolic demands of the 2 exercise modes. Clearly, if these findings can be replicated across other samples, this pattern of results could provide a basis to explore potential mechanisms that may be responsible for acute yoga-induced changes in cognition, using neuroimaging techniques. Future research investigating acute exercise effects on cognition should also examine whether nontraditional modes of exercise such as tai-chi, yoga, and forms of martial arts are equally effective in influencing cognitive processes and the timespan of improved cognitive performance.

\section{References}

1. McMorris T, Tomporowski P, Audiffren M. Exercise and cognitive function. Michigan: Wiley- Blackwell; 2009.

2. Brisswalter J, Collardeau M, Rene' A. Effects of acute physical exercise characteristics on cognitive performance. Sports Med. 2002;32:555-566. PubMed doi:10.2165/00007256-200232090-00002

3. McMorris T, Graydon J. The effect of incremental exercise on cognitive performance. Int J Sport Psychol. 2000;31:66-81.

4. Tomporowski PD. Effects of acute bouts of exercise on cognition. Acta Psychol (Amst). 2003;112:297-324. PubMed doi:10.1016/S0001-6918(02)00134-8

5. Etnier JL, Salazar W, Landers DM, Petruzello SJ, Han M, Nowell P. The influence of physical fitness and exercise upon cognitive functioning: a meta-analysis. J Sport Exer Psychol. 1997;19:249-277.

6. Colcombe S, Kramer AF. Fitness effects on the cognitive function of older adults: a meta-analytic study. Psychol Sci. 2003;14(2):125-130. PubMed doi:10.1111/1467-9280. t01-1-01430

7. Hillman CH, Snook EM, Jerome GJ. Acute cardiovascular exercise and executive control function. Int J Psychophysiol. 2003;48:307-314. PubMed doi:10.1016/S01678760(03)00080-1

8. Ross A, Thomas S. The health benefits of yoga and exercise: a review of comparison studies. J Altern Complement Med. 2010;16(1):3-12. PubMed doi:10.1089/ acm.2009.0044

9. Taimini IK. The Science of Yoga. Madras, India: The Theosophical Publishing House; 1961.

10. Sarang SP, Telles S. Immediate effect of two yoga-based relaxation techniques on performance in a letter-cancellation task. Percept Mot Skills. 2007;105:379-385. PubMed

11. Telles S, Raghuraj P, Arankalle D, Naveen KV. Immediate effect of high frequency yoga breathing on attention. Indian J Med Sci. 2008;62(1):20-22. PubMed doi:10.4103/0019-5359.38919

12. Canter PH, Ernst E. The cumulative effects of Transcendental Meditation on cognitive function-a systematic 
review of randomised controlled trials. Wien Klin Wochenschr. 2003;115(21-22):758-766. PubMed doi:10.1007/ BF03040500

13. Pontifex MB, Hillman CH, Fernhall B, Thompson KM, Valentini TA. The effect of acute aerobic and resistance exercise on working memory. Med Sci Exerc Sport. 2009;41(4):927-934. PubMed doi:10.1249/ MSS.0b013e3181907d69

14. Owen AM, McMillan KM, Laird AR, Bullmore E. N-back working memory paradigm: a meta-analysis of normative functional neuroimaging studies. Hum Brain Mapp. 2005;25:46-59. PubMed doi:10.1002/hbm.20131

15. Eriksen CW, Eriksen BA. Effects of noise letters upon the identification letter in a non-search task. Percept Psychophys. 1974;16:143-149. doi:10.3758/BF03203267

16. Hillman CH, Motl RW, Pontifex MB, et al. Physical activity and cognitive function in a cross-section of younger and older community-dwelling individuals. Health Psychol. 2006;25(6):678-687. PubMed doi:10.1037/02786133.25.6.678

17. Pontifex MB, Hillman CH. Neuroelectric and behavioral indices of interference control during acute cycling. Clin Neurophysiol. 2007;118:570-580. PubMed doi:10.1016/j. clinph.2006.09.029

18. Kirchner WK. Age differences in short-term retention of rapidly changing information. $J$ Exp Psychol. 1958;55(4):352-358. PubMed doi:10.1037/h0043688

19. Nystrom LE, Braver TS, Sabb FW, Delgado MR, Noll DC, Cohen JD. Working memory for letters, shapes and locations: fMRI evidence against stimulus-based regional organization in human prefrontal cortex. Neuroimage. 2000;11:424-446. PubMed doi:10.1006/nimg.2000.0572

20. American College of Sports Medicine. ACSM's guidelines for exercise testing and prescription. 7th ed. New York: Lippincott Williams \& Wilkins; 2006:55-92.
21. Borg G. Perceived exertion as an indicator of somatic stress. Scand J Rehabil Med. 1970;2:92-98. PubMed

22. Tomporowski PD, Cureton K, Armstrong LE, Kane GM, Sparling PB, Millard-Stafford M. Short-term effects of aerobic exercise on executive processes and emotional reactivity. Int J Sport Exerc Psychol. 2005;3:131-146. doi $: 10.1080 / 1612197 X .2005 .9671763$

23. Fletcher PC, Henson RN. Frontal lobes and human memory: insights from functional neuroimaging. Brain. 2001;124:849-881. PubMed doi:10.1093/brain/124.5.849

24. Berger BG, Owen DR. Stress reduction and mood enhancement in four exercise modes: swimming, body conditioning, Hatha yoga, and fencing. Res Q Exerc Sport. 1988;59(2):148-159. doi:10.1080/02701367.1988.10605 493

25. Berger BG, Owen DR. Mood alteration with yoga and swimming: aerobic exercise may not be necessary. Percept Mot Skills. 1992;75(3 Pt 2):1331-1343. PubMed doi:10.2466/pms.1992.75.3f.1331

26. Nardo AC, Reynolds C. Social, emotional, behavioral and cognitive benefits of yoga for children: a nontraditional role for school psychologists to consider. Paper presented at the annual meeting of the National Association of School Psychologists 2002; Chicago, IL.

27. Telles S, Srinivas RB. Autonomic and respiratory measures in children with impaired vision following yoga and physical activity programs. Int J Rehabil Health. 1998;4:117-122. doi:10.1023/A:1022912626238

28. Fox E. Attentional bias in anxiety: selective or not? Behav Res Ther. 1993;31:487-493. PubMed doi:10.1016/0005-7967(93)90129-I 